\title{
Apatinib Combined with Irinotecan in the Treatment of Advanced Small-Cell Esophageal Carcinoma: A Case Report
}

This article was published in the following Dove Press journal: OncoTargets and Therapy

\author{
Longhua Guo' \\ Xiaofang Zou (D) ${ }^{\prime}$ \\ Yinfang Gu' \\ Lilan $\mathrm{Yi}^{\mathrm{I}}$ \\ Jingjing Zhao ${ }^{2}$ \\ Guowu Wu (D)' \\ 'Department of Medical Oncology, \\ Cancer Center, Meizhou People's \\ Hospital (Huangtang Hospital), Meizhou \\ Hospital Affiliated to Sun Yat-Sen \\ University, Meizhou, 5I403I, People's \\ Republic of China; ${ }^{2}$ Department of \\ Biotherapy, Sun Yat-Sen University \\ Cancer Center, State Key Laboratory of \\ Oncology in South China, Collaborative \\ Innovation Center for Cancer Medicine, \\ Guangzhou, 510060, People's Republic of \\ China
}

Correspondence: Jingjing Zhao State Key Laboratory of Oncology in South China; Department of Biotherapy, Sun Yat-Sen University Cancer Center, 65I Dongfeng Road East, Guangzhou, 510060, People's Republic of China

Tel +86-20-87345699

Fax +86-20-87343392

Email zhaojingj@sysucc.org.cn

Correspondence: Guowu Wu Department of Medical Oncology, Cancer Center, Meizhou People's Hospital (Huangtang Hospital), Meizhou Hospital Affiliated to Sun Yat-Sen University, 63

Huangtang Road, Meizhou, 5I403I,

People's Republic of China

Tel +86-753-2I31682

Fax +86-753-2204840

Email guowuwugd@163.com

\begin{abstract}
Chemotherapy is the mainstay of treatment for advanced small cell esophageal carcinoma (SCEC) characterized by poor prognosis. Preclinical studies demonstrated that apatinib has the potential to enhance the efficacy of conventional chemotherapeutic drugs and reverse multidrug resistance (MDR). This report described the application of apatinib combined with irinotecan as the third-line treatment for advanced SCEC in a 54-year-old male patient. His symptoms of upper abdominal pain and distension were ameliorated notably after the combination therapy. Computed tomography (CT) examination revealed the treatment efficacy was partial response (PR). The progression-free survival (PFS) and overall survival (OS) were 12.5 months and 28 months, respectively. The treatment-related toxicity was manageable. Apatinib combined with chemotherapy may serve as a new treatment choice for advanced SCEC patients. However, further studies should be conducted to confirm the therapeutic value of this combination regimen in advanced SCEC.
\end{abstract}

Keywords: small cell esophageal carcinoma, apatinib, irinotecan, progression-free survival, overall survival

\section{Introduction}

Small cell esophageal carcinoma (SCEC) is an extrapulmonary small cell carcinoma accounting for approximately $0.5-2.8 \%$ of all primary esophageal malignancies. ${ }^{1-3}$ This disease is characterized by high malignancy, widespread metastasis at diagnosis, and poor prognosis. ${ }^{4}$ In the advanced stage, chemotherapy strategy is similar to that for small cell lung cancer (SCLC), including cisplatin (CDDP) plus etoposide (VP16), CDDP plus irinotecan (CPT-11), and taxanes. ${ }^{5}$ However, patients with widespread metastatic SCEC will relapse from chemotherapy rapidly. Novel therapeutic approaches are needed to improve the survival of these patients.

Apatinib (Hengrui Pharmaceutical Co. Ltd, Jiangsu, People's Republic of China) is a small-molecule tyrosine kinase inhibitor and novel antiangiogenic agent, specifically targeting the vascular endothelial growth factor receptor-2 (VEGFR-2). It presents encouraging efficacy in the treatment of a variety of solid tumors, such as advanced gastric cancer, breast cancer, hepatocellular carcinoma, and non-small cell lung cancer (NSCLC). ${ }^{6}$ Now this targeted drug has been approved for the second- or subsequent-line treatment of advanced gastric or gastroesophageal junction adenocarcinoma in China based on two prospective clinical trials. $^{7,8}$ 
In this report, we presented a case of advanced relapsed SCEC refractory to second-line treatment unexpectedly responded to the combination therapy of apatinib plus irinotecan.

\section{Case Report}

A 54-year-old male mainly complained of upper abdominal pain and distension was referred to Sun Yatsen University Cancer Center in July 2016. The endoscopic biopsy of an esophageal tumor specimen indicated a poorly differentiated cancer, and immunohis tochemistry (IHC) showed it was small cell esophageal carcinoma with Syn (+), CD56 (+), CK (+), NSE (+), CgA (-), CK5/6 (-), P63 (-), and P40 (-) (Figure 1). The patient was diagnosed with advanced SCEC (cT3N3M1, stage IV) by contrast-enhanced computed tomography (CT) (Figure 2) and ultrasound gastroscopy (Figure 3). Six cycles of EP regimen (etoposide and cisplatin) were administered as the first-line chemotherapy and the PFS was about 8 months. From September 1, 2017, he received a second-line chemotherapy of paclitaxel $\left(80 \mathrm{mg} / \mathrm{m}^{2}\right.$, day $\left.1,8,15\right)$, whereas this treatment was not effective and the PFS was only one month.

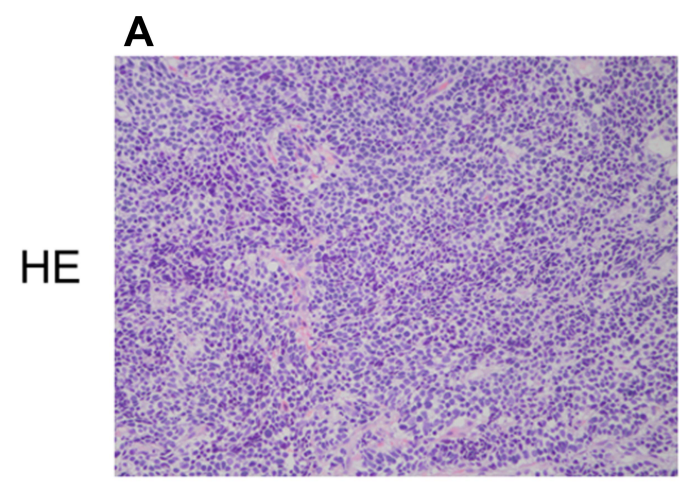

\section{B}

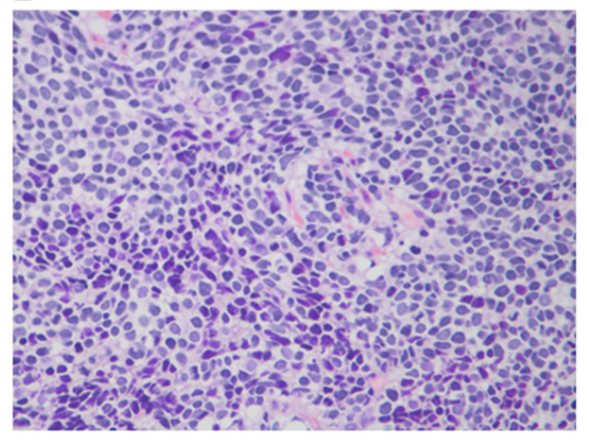

D

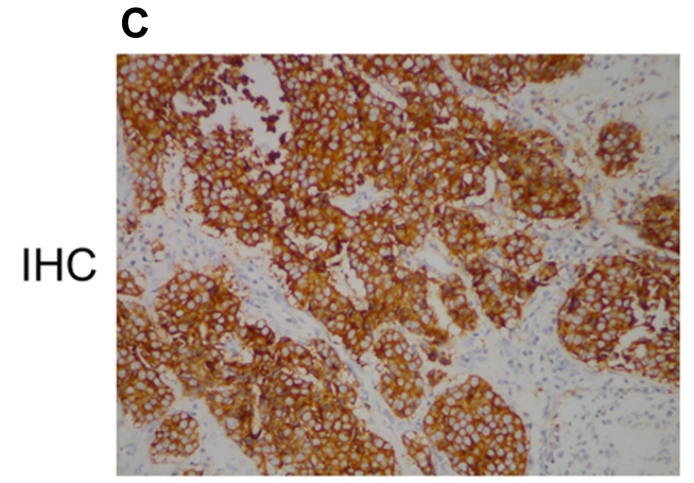

Syn (+)

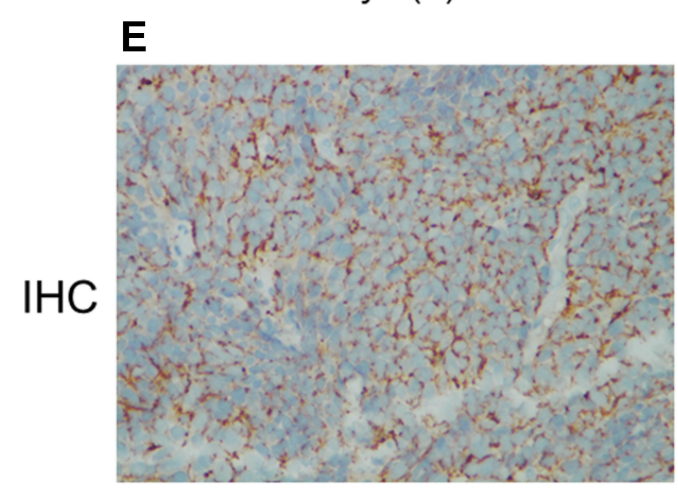

CK $(+)$

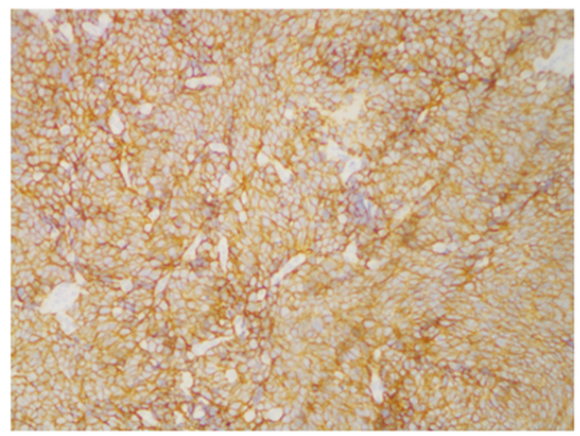

CD56 (+)

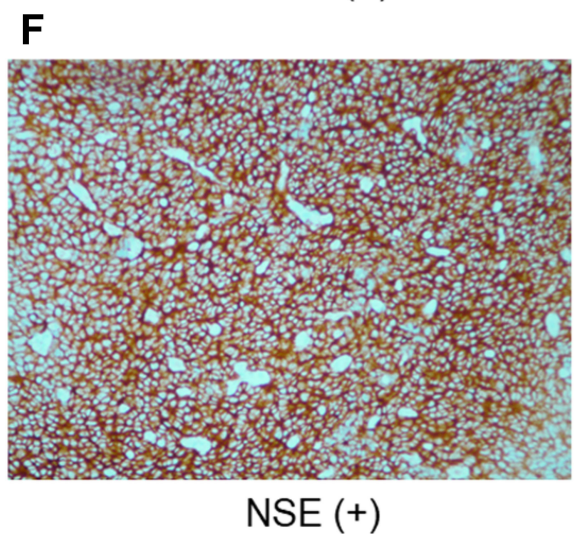

Figure I Pathological images of the esophageal mass by endoscopic biopsy. (A) Hematoxylin-eosin stain $(\times 200)$. (B) Hematoxylin-eosin stain $(\times 400)$. (C-F) Immunohistochemical staining $(\times 200)$ : Syn $(+)$, CD56 (+), CK $(+)$, NSE (+). 
A

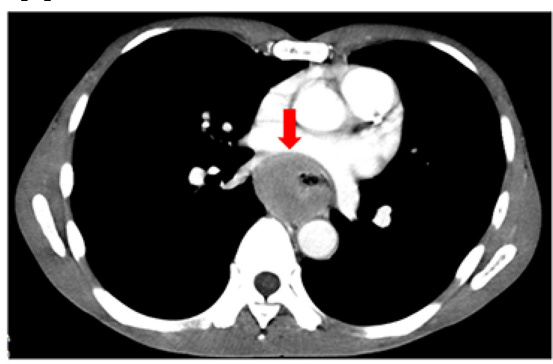

D

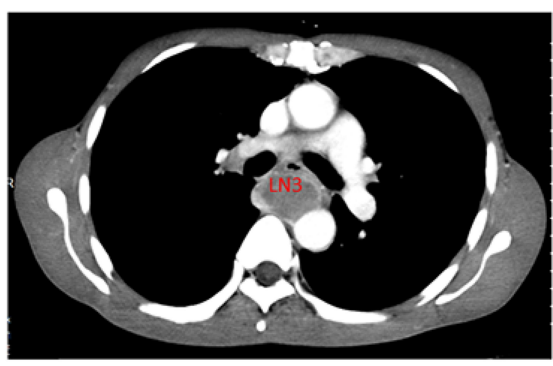

G

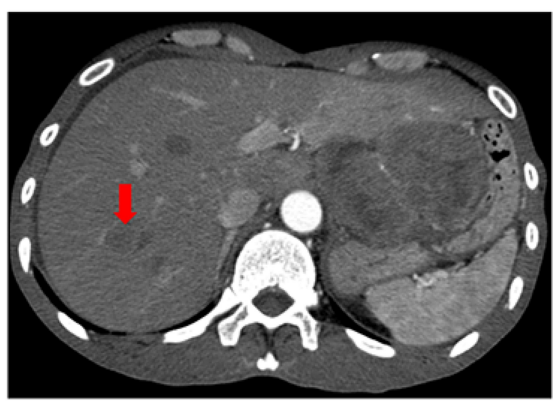

B

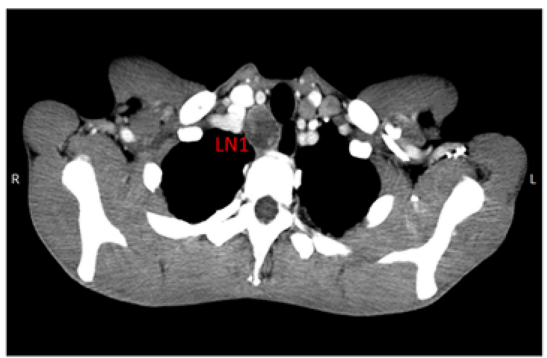

E

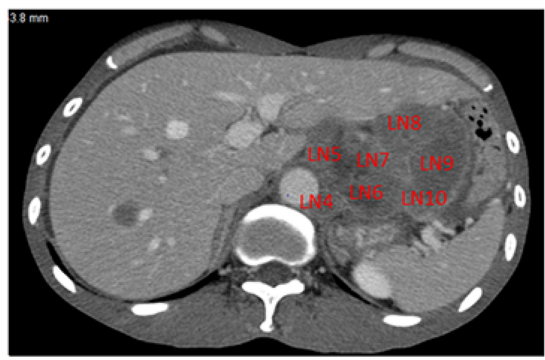

H

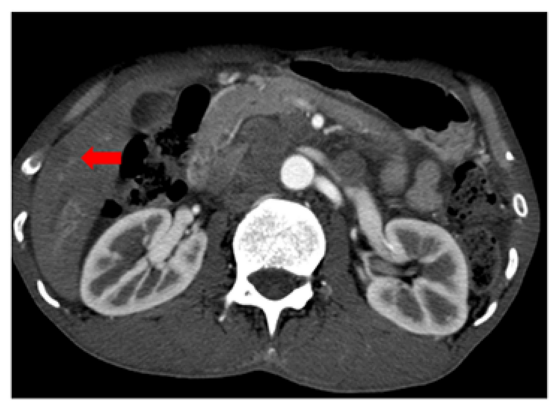

C

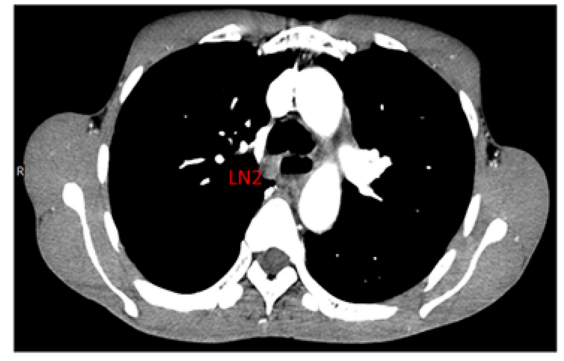

F

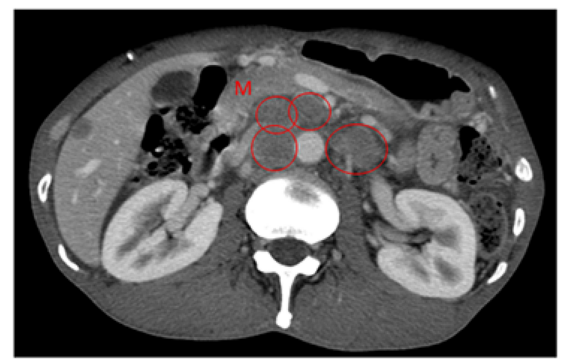

I

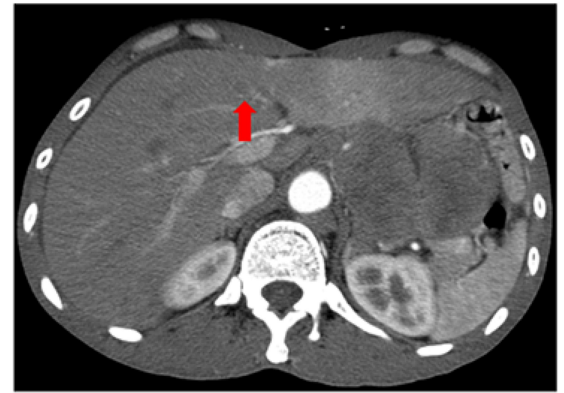

Figure 2 Chest and abdominal contrast-enhanced CT images at diagnosis. (A) Primary esophageal tumor in the middle/lower third of the esophagus. As can be seen at the red arrow, the wall of the esophagus was thickened, with the whole layer being infiltrated, and the boundary was clear, which means T3 according to TNM stage criteria of the Union for International Cancer Control (UICC). (B-E) Regional enlarged lymph nodes (LN): more than seven regional metastatic lymph nodes means N3 according to TNM stage criteria of UICC. (F) The red circles indicated metastatic lymph nodes in the abdominal cavity and retroperitoneum (M: metastasis). (G-I) The red arrow indicated liver metastatic nodes.

In October 2017, the patient visited Meizhou People's Hospital (Huangtang Hospital) and underwent CT scans (Figure 4A1-A3). The patient could smoothly eat semiliquid diet with slight sense of obstruction, but the main complain was abdominal distension after eating.

However, no standard third-line medications were available for advanced SCEC even for advanced stage SCLC. According to the studies on esophageal carcinoma and smallcell lung cancer, ${ }^{9,10}$ chemotherapy with irinotecan (CPT-11) might be the optimal treatment strategy for this patient, however, continued chemotherapy is likely to result in rapid resistance. At that time, the targeted drug apatinib has been approved for the second- or subsequent-line treatment of advanced gastric cancer. ${ }^{8}$ In addition, preclinical studies demonstrated that apatinib might enhance the efficacy of conventional chemotherapeutic drugs and reverse multidrug resistance (MDR). ${ }^{11}$ Therefore, the patient was recommended to try irinotecan combined with apatinib. We communicated fully with the patient and his family regarding the off-label use of apatinib and the risks for potential bleeding and rupture of the esophageal lesion before they signed the consent form. Subsequently, apatinib (500 mg, oral, daily) combined with irinotecan $\left(65 \mathrm{mg} / \mathrm{m}^{2}, \mathrm{~d} 1, \mathrm{~d} 8\right.$, every three weeks) was administered as the third-line treatment until the progression of the disease. Although the patients did not complain of obvious dysphagia, he was advised to grind apatinib into powder and take it in fluid state in the beginning of the treatment for the risk of esophageal blockage according to CT scans. His symptoms of upper abdominal pain and distension were ameliorated notably after six weeks of treatment. The measurable lesion in 
A
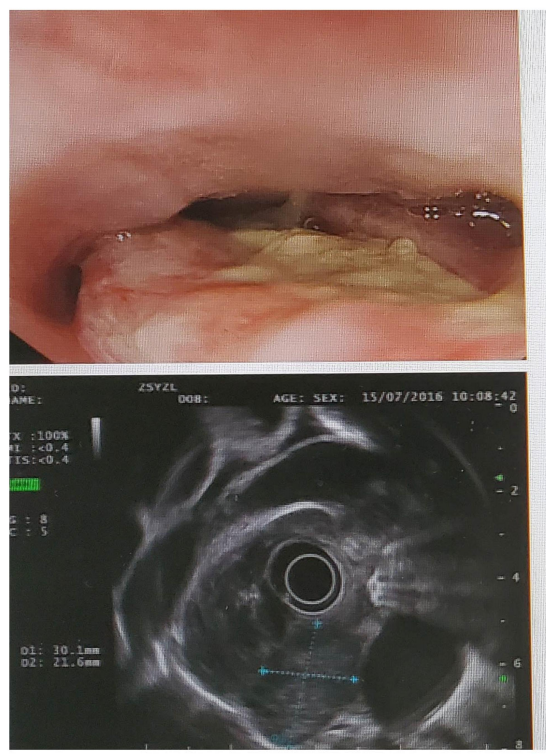

D
B

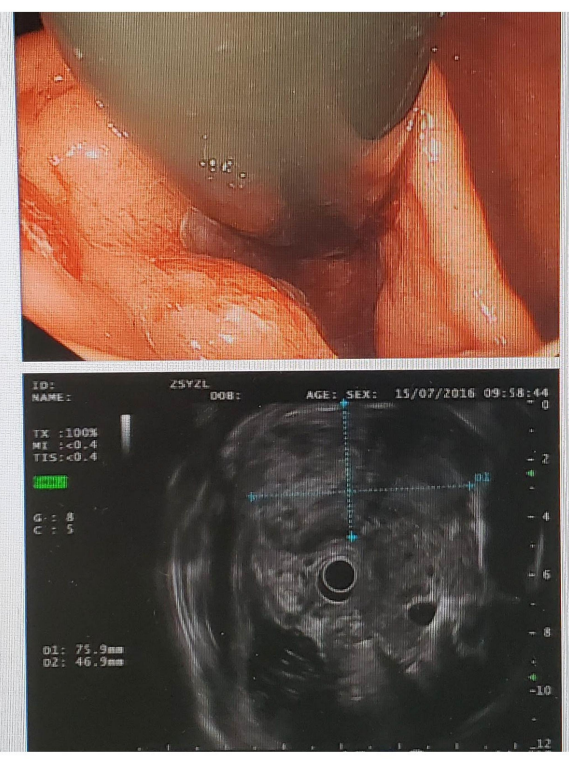

E
C
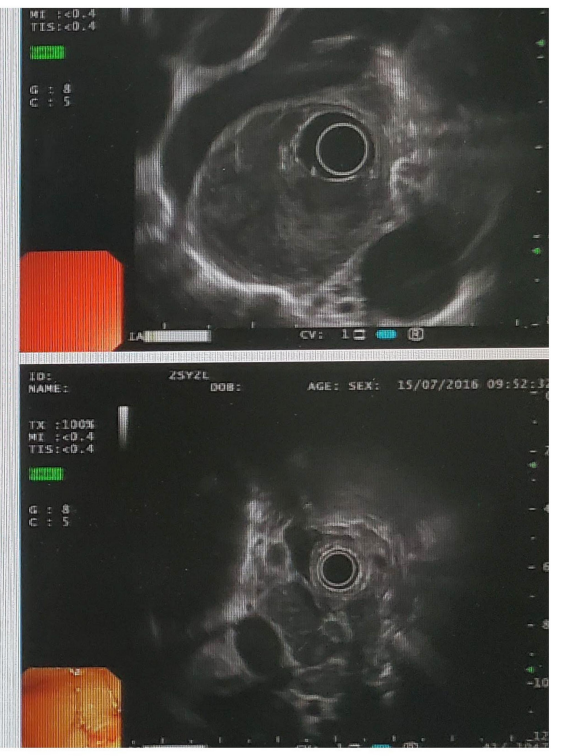

$\mathbf{F}$

Figure 3 The images of ultrasound gastroscopy. (A) Esophageal tumor. (B) Cardia tumor. (C) Esophageal hypoechoic mass. (D) Swollen and hypoechoic lymph nodes beside esophagus. (E) Hypoechoic mass in abdominal cavity. (F) Multiple swollen and hypoechoic lymph nodes in retroperitoneum.

the esophagus and liver significantly shrunk and the optimal response was partial response (PR) according to the Response Evaluation Criteria in Solid Tumors (RECIST) 1.1 standard (Figure 4B1-B3). The patient was then suggested to take apatinib by swallowing it directly. This combination regimen was continuously used as maintenance therapy. His disease progressed on October 15, 2018, achieving a PFS of about 12.5 months (Figure 4C1-C3). He deceased on November 29, 2018 , because of systemic failure. The overall survival (OS) was 28 months. The minor adverse events (AEs) due to the combination treatment were tolerable grade 2 hand-foot syndrome and a decrease in the number of leukocytes and granulocytes.

\section{Discussion}

SCEC behaves aggressively and $40-60 \%$ of the SCEC patients had widespread metastases at the time of diagnosis. ${ }^{4}$ Because of its rarity, the standard treatment has not yet been established. Chemotherapy is the key therapeutic method for patients with advanced SCEC on the basis of retrospective studies and the experience from SCLC treatment. ${ }^{2,12}$ Although SCEC is sensitive to initial chemotherapy, rapid drug resistance makes the post-first-line treatment a big challenge. Previous studies indicated that advanced SCEC patients had a very poor prognosis, with their median survival ranging from 4.9 to 8.5 months postchemotherapy. ${ }^{2,12-15}$ In this report, the therapeutic regimen of apatinib combined with irinotecan was applied in the advanced
SCEC patient after the failure of second-line chemotherapy. The PFS achieved 12.5 months and the OS extended to 28 months. Treatment-related AEs were tolerable. To the best of our knowledge, this is the first report concerning the application of apatinib combined with chemotherapy in the post firstline therapy of advanced SCEC.

Angiogenesis plays a critical role in tumor growth, progression, and metastatic spread. Vascular endothelial growth factor (VEGF) is a major driver of tumor angiogenesis expressed in various tumor types that lead to poor survival. ${ }^{16,17}$ It has been demonstrated that anti-VEGF agents increase the intra-tumor chemotherapeutic drug levels by normalizing the disorganized and dysfunctional tumor vasculature and improve the delivery of the drug to the tumor center where the pressure is maximal by reducing the high interstitial fluid pressure of the tumor. ${ }^{18,19}$ For instance, bevacizumab, one of the antiangiogenic agents, has been approved to combine with carboplatin plus paclitaxel as a first-line therapeutic strategy in patients with advanced non-squamous NSCLC by Food and Drug Administration (FDA). ${ }^{20}$ Apatinib is a novel and selective inhibitor of VEGFR-2 tyrosine kinase exhibiting broadspectrum antitumor activities. ${ }^{21}$ In a preclinical study, apatinib has been proved to enhance the efficacy of conventional chemotherapeutical drugs in the side population as well as ABCB1-overexpressing leukemia cells and strongly reverse the MDR in K562/ADR cells. ${ }^{11,22}$ Another study indicated that apatinib treatment could significantly increase the 

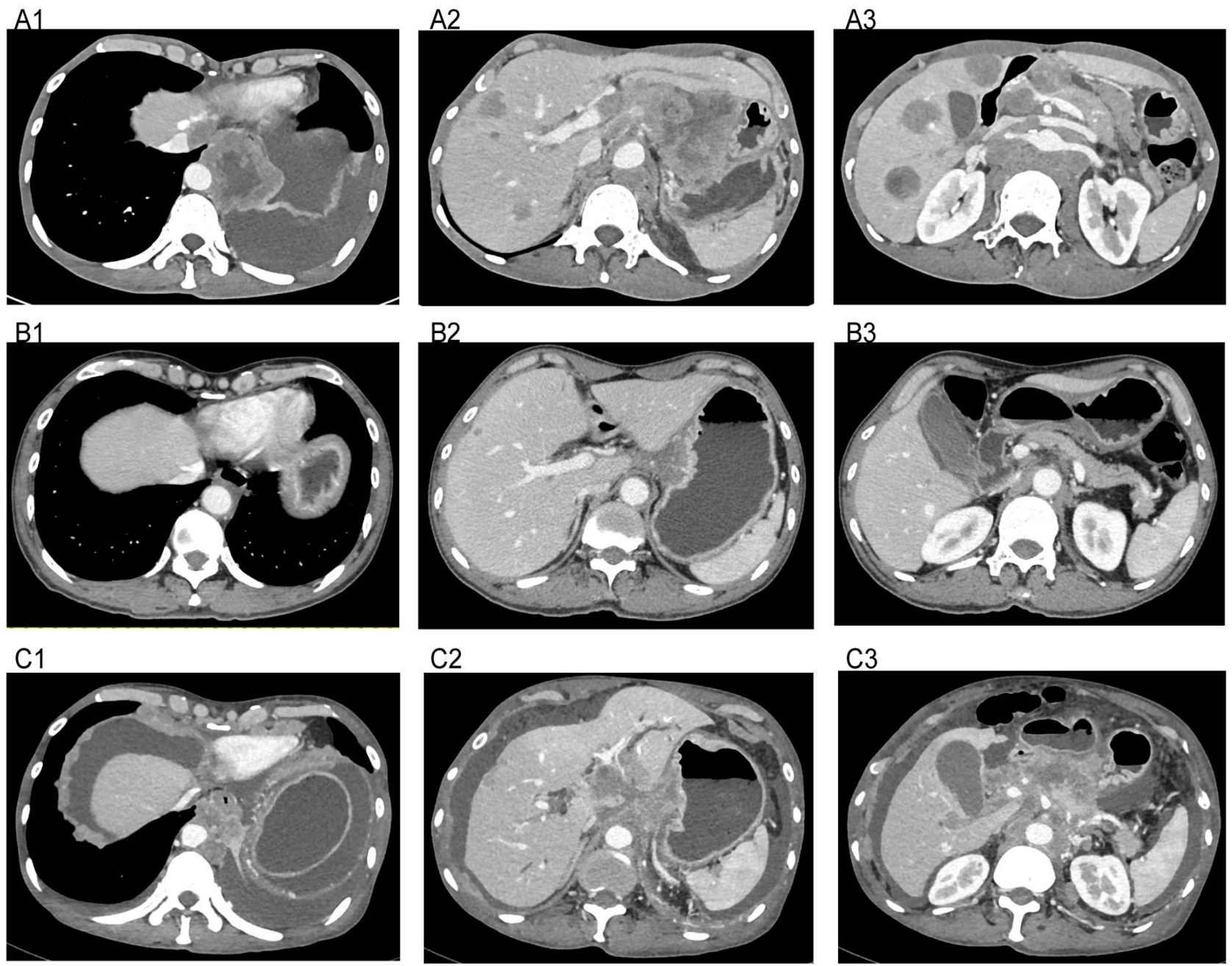

Figure 4 Computed tomography images of the treatment with apatinib plus irinotecan. (AI-A3) Before the treatment of apatinib and irinotecan on September $30,2017$. (BI-B3) Efficacy of apatinib and irinotecan treatment on May 14, 20I8. (CI-C3) Disease progressed after apatinib plus irinotecan treatment on October I5, 2018.

concentration of docetaxel in A549 xenograft nude mice. ${ }^{23}$ In terms of clinical studies, the promising antitumor efficacy of apatinib was demonstrated in advanced stage SCLC, the histological type of which is similar to SCEC. ${ }^{24}$ For example, a randomized Phase II study of extensive-stage SCLC showed that patients treated with apatinib in combination with chemotherapy had a median PFS of 7.8 months and a medium OS of 12.1 months, which were significantly longer than those in patients treated with chemotherapy alone. ${ }^{25}$ A previous retrospective study presented that induction chemotherapy followed by apatinib maintenance therapy achieved a median PFS of 8.3 months (95\% CI: $7.20-9.40$ months) and a median OS of 12.5 months (95\% CI: 5.51-19.49 months) in advanced stage SCLC. ${ }^{26}$ These results indicated that apatinib was an encouraging treatment option for advanced SCLC despite the rarity of large-sample clinical trials. Up to now, apatinib combined with chemotherapy in the treatment of advanced SCEC was rarely reported. In our report, the patient with advanced SCEC received apatinib combined with irinotecan as the third-line treatment. After the combination therapy, the patient's symptoms of upper abdominal pain and distension were relieved considerably and the best response achieved was PR. Eventually, the PFS was 12.5 months and OS extended to 28 months, which were longer than those reported in previous studies. This case report further demonstrated the encouraging clinical efficacy of the regimen of apatinib combined with chemotherapeutic drugs on solid tumors, even for SCEC. Nevertheless, more evidence is needed to confirm the clinical benefit of this combination regimen in advanced SCEC treatment.

\section{Ethics and Consent Statement}

Ethics approval of this case report was granted by the Institutional Ethics Review Board of Meizhou People's 
Hospital (Huangtang Hospital). Written informed consent regarding the risks associated with drug therapy was obtained from the patient before the treatment.

\section{Consent for Publication}

Written informed consent was obtained from the patient for publication of this case report. Institutional approval was not required to publish the case details.

\section{Author Contributions}

All authors made substantial contributions to conception and design, acquisition of data, or analysis and interpretation of data; took part in drafting the article or revising it critically for important intellectual content; agreed to submit to the current journal; gave final approval of the version to be published; and agree to be accountable for all aspects of the work.

\section{Disclosure}

The authors report no conflicts of interest in this study.

\section{References}

1. Chen WW, Wang F, Chen S, et al. Detailed analysis of prognostic factors in primary esophageal small cell carcinoma. Ann Thorac Surg. 2014;97(6):1975-1981. doi:10.1016/j.athoracsur.2014.02.037

2. Wong AT, Shao M, Rineer J, Osborn V, Schwartz D, Schreiber D. Treatment and survival outcomes of small cell carcinoma of the esophagus: an analysis of the National Cancer Data Base. Dis Esophagus. 2017;30(2):1-5. doi:10.1111/dote.12487

3. Xu L, Li Y, Liu X, et al. Treatment strategies and prognostic factors of limited-stage primary small cell carcinoma of the esophagus. $J$ Thorac Oncol. 2017;12(12):1834-1844. doi:10.1016/j.jtho.2017.09.1966

4. Lv J, Liang J, Wang J, et al. Primary small cell carcinoma of the esophagus. J Thorac Oncol. 2008;3(12):1460-1465. doi:10.1097/ JTO.0b013e31818e1247

5. Xiao Q, Xiao H, Ouyang S, Tang J, Zhang B, Wang H. Primary small cell carcinoma of the esophagus: comparison between a Chinese cohort and Surveillance, Epidemiology, and End Results (SEER) data. Cancer Med. 2019;8:1074-1085. doi:10.1002/cam4.2001

6. Scott LJ. Apatinib: a review in advanced gastric cancer and other advanced cancers. Drugs. 2018;78(7):747-758. doi:10.1007/s40265-018-0903-9

7. Li J, Qin S, Xu J, et al. Apatinib for chemotherapy-refractory advanced metastatic gastric cancer: results from a randomized, placebo-controlled, parallel-arm, phase II trial. J Clin Oncol. 2013;31(26):3219-3225. doi:10.1200/JCO.2013.48.8585

8. Li J, Qin S, Xu J, et al. Randomized, double-blind, placebo-controlled phase III trial of apatinib in patients with chemotherapy-refractory advanced or metastatic adenocarcinoma of the stomach or gastroesophageal junction. J Clin Oncol. 2016;34(13):1448-1454. doi:10.1200/JCO.2015.63.5995

9. Wang $X$, Wang X, Huang J. Irinotecan plus fluorouracil-based regimen as second or third-line chemotherapy for recurrent or metastatic esophageal squamous cell carcinoma. Thorac Cancer. 2016;7(2):246-250. doi:10.1111/1759-7714.12323
10. Noda K, Nishiwaki Y, Kawahara M, et al. Irinotecan plus cisplatin compared with etoposide plus cisplatin for extensive small-cell lung cancer. NEngl J Med. 2002;346(2):85-91. doi:10.1056/NEJMoa003034

11. Mi YJ, Liang YJ, Huang HB, et al. Apatinib (YN968D1) reverses multidrug resistance by inhibiting the efflux function of multiple ATP-binding cassette transporters. Cancer Res. 2010;70 (20):7981-7991. doi:10.1158/0008-5472.CAN-10-0111

12. Lu XJ, Luo JD, Ling Y, et al. Management of small cell carcinoma of esophagus in China. J Gastrointest Surg. 2013;17(7):1181-1187. doi:10.1007/s11605-013-2204-7

13. Al Mansoor S, Ziske C, Schmidt-Wolf IG. Primary small cell carcinoma of the esophagus: patient data metaanalysis and review of the literature. Ger Med Sci. 2013;11:Doc12. doi:10.3205/000180

14. Vos B, Rozema T, Miller RC, et al. Small cell carcinoma of the esophagus: a multicentre Rare Cancer Network study. Dis Esophagus. 2011;24 (4):258-264. doi:10.1111/j.1442-2050.2010.01133.x

15. Kukar M, Groman A, Malhotra U, et al. Small cell carcinoma of the esophagus: a SEER database analysis. Ann Surg Oncol. 2013;20 (13):4239-4244. doi:10.1111/j.1442-2050.2010.01133.x

16. Folkman J. Tumor angiogenesis: therapeutic implications. $N$ Engl J Med. 1971;285(21):1182-1186. doi:10.1056/ NEJM197111182852108

17. McMahon G. VEGF receptor signaling in tumor angiogenesis. Oncologist. 2000;5(Suppl 1):3-10. doi:10.1634/theoncologist.5-suppl_1-3

18. Goel S, Duda DG, Xu L, et al. Normalization of the vasculature for treatment of cancer and other diseases. Physiol Rev. 2011;91 (3):1071-1121. doi:10.1152/physrev.00038.2010

19. Jain RK. Antiangiogenesis strategies revisited: from starving tumors to alleviating hypoxia. Cancer Cell. 2014;26(5):605-622. doi:10.1016/j.ccell.2014.10.006

20. Zhou C, Wu YL, Chen G, et al. BEYOND: a randomized, double-blind, placebo-controlled, multicenter, phase study of III first-line carboplatin/paclitaxel plus bevacizumab or placebo in Chinese patients with advanced or recurrent nonsquamous non-smallcell lung cancer. J Clin Oncol. 2015;33(19):2197-2204. doi:10.1200/ JCO.2014.59.4424

21. Tian S, Quan H, Xie C, et al. YN968D1 is a novel and selective inhibitor of vascular endothelial growth factor receptor-2 tyrosine kinase with potent activity in vitro and in vivo. Cancer Sci. 2011;102(7):1374-1380. doi:10.1111/j.1349-7006.2011.01939.x

22. Tong XZ, Wang F, Liang S, et al. Apatinib (YN968D1) enhances the efficacy of conventional chemotherapeutical drugs in side population cells and $\mathrm{ABCB} 1$-overexpressing leukemia cells. Biochem Pharmacol. 2012;83(5):586-597. doi:10.1016/j.bcp.2011.12.007

23. Feng SQ, Wang GJ, Zhang JW, et al. Combined treatment with apatinib and docetaxel in A549 xenograft mice and its cellular pharmacokinetic basis. Acta Pharmacol Sin. 2018;39(10):1670-1680. doi:10.1038/aps.2018.16

24. Xu Y, Huang Z, Lu H, et al. Apatinib in patients with extensive-stage small-cell lung cancer after second-line or third-line chemotherapy: a Phase II, single-arm, multicentre, prospective study. $\mathrm{Br} J$ Cancer. 2019;121:640-646. doi:10.1038/s41416-019-0583-6

25. Luo H, Zhang L, Yang B, et al. A randomized Phase 2 trial of apatinib vs observation as maintenance treatment following first-line induction chemotherapy in extensive-stage small cell lung cancer. Invest New Drugs. 2019;38:148-159. doi:10.1007/s10637019-00828-x

26. Yan X, Wang Q, Wang H, et al. Apatinib as maintenance therapy in extensive-stage small-cell lung cancer: results from a single-center retrospective study. J Cancer Res Clin Oncol. 2019;145(1):235-240. doi:10.1007/s00432-018-2764-8 


\section{Publish your work in this journal}

OncoTargets and Therapy is an international, peer-reviewed, open access journal focusing on the pathological basis of all cancers, potential targets for therapy and treatment protocols employed to improve the management of cancer patients. The journal also focuses on the impact of management programs and new therapeutic

Submit your manuscript here: https://www.dovepress.com/oncotargets-and-therapy-journal agents and protocols on patient perspectives such as quality of life, adherence and satisfaction. The manuscript management system is completely online and includes a very quick and fair peer-review system, which is all easy to use. Visit http://www.dovepress.com/ testimonials.php to read real quotes from published authors. 